\title{
AN UNUSUAL CASE OF HERLYN WERNER WUNDERLICH SYNDROME PRESENTING DURING PREGNANCY: A CASE REPORT
}

Priyanka Priyadarshini Baishya ${ }^{1}$, Deb Kumar Boruah², Chang Hom Thoumoung ${ }^{3}$, Dhaval Dhingahi ${ }^{4}$

\section{HOW TO CITE THIS ARTICLE:}

Priyanka Priyadarshini Baishya, Deb Kumar Boruah, Chang Hom Thoumoung, Dhaval Dhingahi. "An Unusual Case of Herlyn Werner Wunderlich Syndrome Presenting During Pregnancy: A Case Report.". Journal of Evolution of Medical and Dental Sciences 2015; Vol. 4, Issue 44, June 01; Page: 7699-7703,

DOI: $10.14260 /$ jemds/2015/1120

ABSTRACT: INTRODUCTION: Herlyn-Werner-Wunderlich syndrome (HWWS), characterized by uterus didelphys, obstructed hemivagina, and ipsilateral renal agenesis, is a rare combined Mullerian and mesonephric duct anomaly. It usually presents after menarche with remittent pelvic pain, dysmenorrhoea or palpable pelvic mass due to hematocolpos. Its presentation during adulthood is rare,(1) and in pregnancy is even rarer.(2) It is one of the causes of infertility. USG \& MRI plays a key role in early diagnosis and prevention of further complications. We present a rare case of Herlyn Werner Wunderlich syndrome in which the diagnosis was delayed until pregnancy.

CASE REPORT: A 25 years old female patient presented with pain abdomen at 12 weeks of pregnancy. She had a previous history of spontaneous abortion at 14 weeks of pregnancy. She also had history of on and off pain abdomen. Because of her bad obstetric history, she was advised an obstetric \& abdominal USG.

USG was done which revealed two separate uterine cavities with well-developed endometrium and myometrium (Fig. 1A). There were two separate cervices in continuity with the two uteri. There was a collection in the vagina (Fig. 1B). Intrauterine pregnancy corresponding to gestational age of 10 weeks was seen in the left horn of the didelphic uterus. Both the ovaries and adnexa were normal. On abdominal USG, the right renal fossa was empty and no evidence of an ectopic right kidney was found. The left kidney was located in the left renal fossa and was bulky due to compensatory hypertrophy.

Further evaluation with MRI was done in a 1.5 Tesla Siemens MR scanner. MRI images showed two uterine horns that were completely separate and widely splayed. Both the horns were fully developed and normal in size, with preserved endometrium and myometrium (Fig. 2A). Early intrauterine pregnancy was present in the left horn (Fig. 2B). Two separate cervices were in continuity with the uterine horns. A longitudinal vaginal septum divided the upper vagina into two cavities, with one cervix entering each hemivagina (Fig. 3A, B). Mildly T1-hyperintense and T2hyperintense collection was present in the right hemivagina (Fig. 3C). The left hemivagina was normal. Both the ovaries were normal. Coronal images through the midabdomen showed an empty right renal fossa and a normal left kidney (Fig. 3A).

These imaging features were diagnostic of HWW syndrome with an intrauterine pregnancy in the left horn with possible incomplete longitudinal vaginal septum, resulting in partial spontaneous decompression of the right hemivagina, which explains the delay in diagnosis until pregnancy. However fetal MRI was not done.

DISCUSSION: The Mullerian ducts develop from embryonic mesoderm cephalocaudally to form the fallopian tubes and fuse distally to create the uterus, cervix, and upper vagina. The development 
occurs approximately between the 6th and the 22nd weeks of gestation.[3] The non-development or partial/complete non-fusion of the Mullerian ducts results in various kinds of Mullerian duct anomalies. Although any type of MDA can be found with renal anomalies embryologically, an increased association in patients with uterus didelphys compared with other types of MDAs was reported.(4)

Herlyn-Werner-Wunderlich syndrome (HWWS) is a triad of uterine didelphys, hemivaginal septum and ipsilateral renal agenesis. In all of the cases with HWW syndrome, renal agenesis was located on the same side with the obstruction, and the right side is twice more common than the left. $(5,6)$ The longitudinal vaginal septum is due to incomplete disappearance of the partition between the fused Müllerian ducts.[7] As an obstructing vaginal malformation, this condition precludes the outflow of menstruation, resulting in hematocolpos, hematometra, and hematosalpinx. The diagnosis is therefore usually made soon after menarche due to symptoms related to the obstructed hemivagina, such as pelvic pain, dysmenorrhoea and a palpable pelvic mass. Long-term complications of HWW syndrome includes endometriosis from retrograde menstruation leading to infertility, and obstetric complications such as recurrent pregnancy loss, preterm labour and prematurity due to uterine anomalies.[8-10]

There are few reported cases where defect in the septum has resulted in spontaneous decompression of the obstructed hemivagina and menstrual blood is discharged from the obstructed hemivagina to the normal side, which may delay presentation of the symptoms. ${ }^{(5,11)}$ Such patients are likely to present with fewer obstructive symptoms than in those with an intact longitudinal vaginal septum. Although some amount of hematocolpos and/or hematometra may be present at diagnosis, the volume may not be sufficient to cause obstructive symptoms.[11] This would explain the delay in presentation as in the case presented. These patients may be protected from retrograde menstruation and subsequent development of pelvic endometriosis and infertility, since the hemivagina is partially decompressed via the defect. This explains preserved fertility and the occurrence of pregnancy in our case.

There are reports of cases of uncomplicated full term pregnancy in uterus didelphys.(12) Reproductive and gestational performances of women with uterus didelphys are well preserved, unless the patient had endometriosis.(13) The pregnancy in a functional hemi uterus has a better prognosis with regard to the fetal survival rate than pregnancy in bicornuate, septate or arcuate uterus.(14)

In our case, the diagnosis of HWW syndrome was delayed until pregnancy. Possibly this patient had a congenital defect in the longitudinal vaginal septum, resulting in partial spontaneous decompression of right-sided obstructed hemivagina and uterine horn till the age of 25 years. Also fertility was preserved in this patient as she did not have any complications related to retrograde menstruation.

CONCLUSION: Herlyn-Werner-Wunderlich syndrome (HWWS) is often a misdiagnosed entity and high index of suspicion is required in patients with Mullerian and mesonephric duct anomalies. It usually presents shortly after puberty. We report a rare case of HWWS, in which the diagnosis was delayed until pregnancy. This patient possibly had a fistulous communication between the two hemivagina and the menstrual blood is discharged from the obstructed to the non-obstructed side.

This explains the delay in presentation of symptoms and also preserved fertility in our case. 


\section{CASE REPORT}

Fig. 1A, 1B: USG images showing two separate uterine horns with well-developed endometrium and myometrium (A) and a collection in the vagina (B).

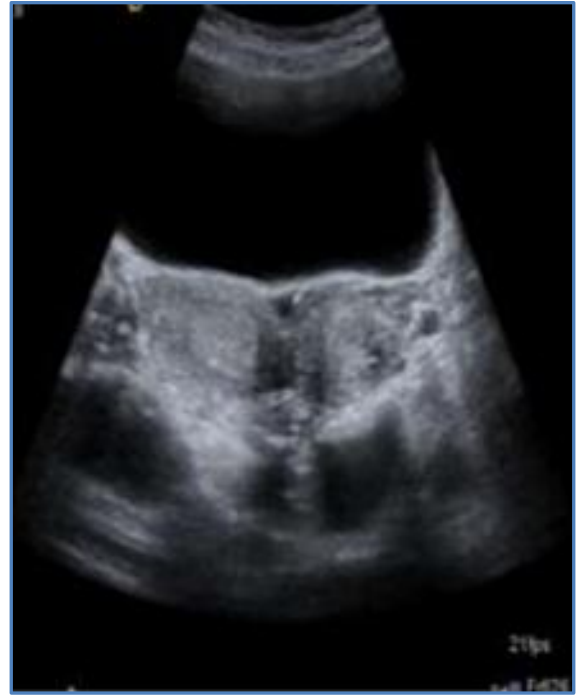

Fig. $1 \mathrm{~A}$

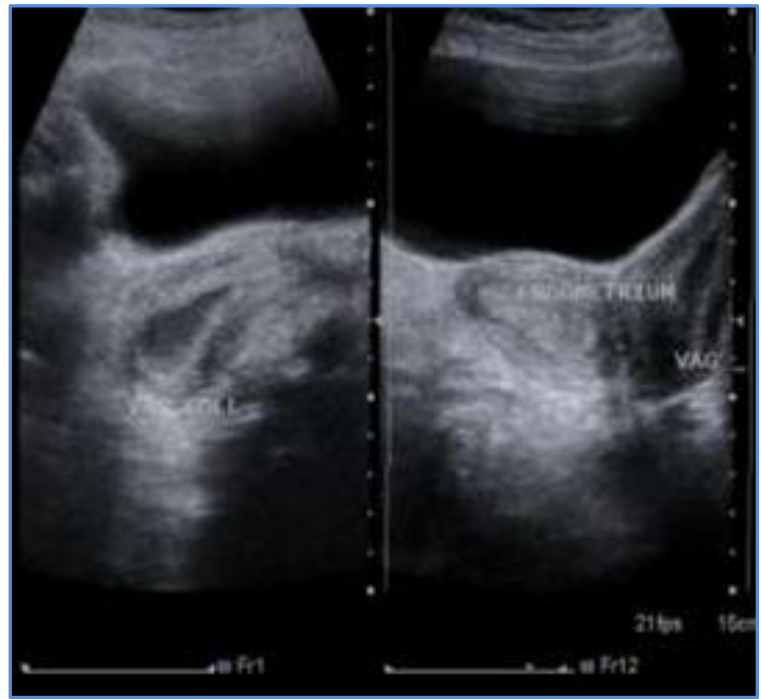

Fig. 1B

Fig. 2A, 2B: Coronal and Axial T2FS images showing two uterine completely separate uterine horns with fully developed endometrium and myometrium(blue arrows). Thick white arrow shows intrauterine pregnancy within the left horn.

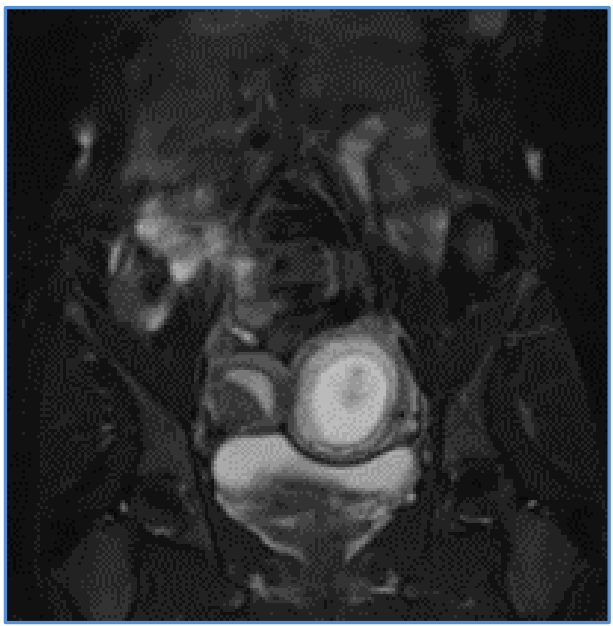

Fig. 2A

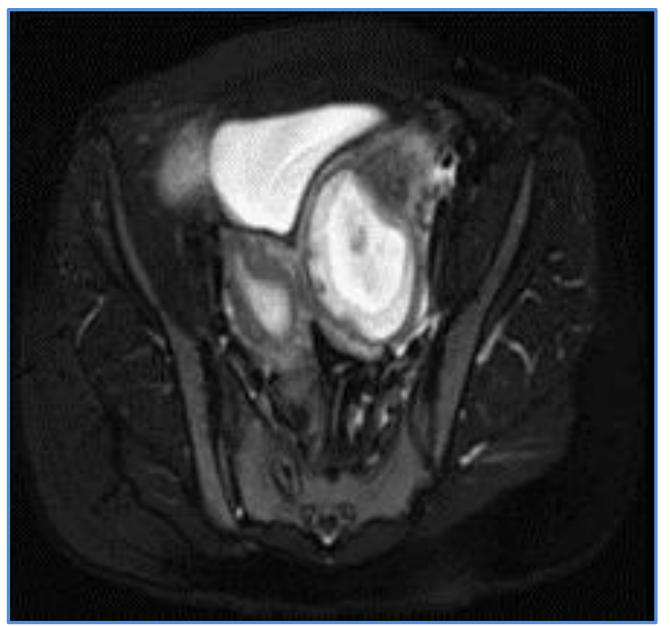

Fig. 2B 


\section{CASE REPORT}

Fig. 3A, 3B: Coronal and Axial T2FS images showing two separate cervices entering into two hemivagina (Blue arrow). Coronal image shows empty right renal fossa (Thick white arrow).

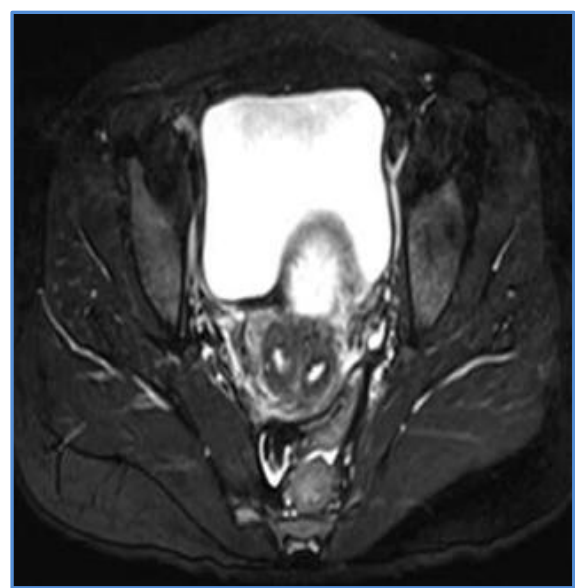

Fig. 3A

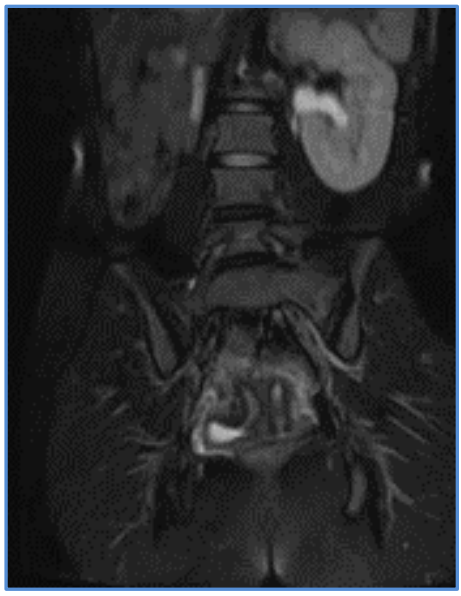

Fig. 3B

Fig. 3C: Axial T1FS image shows T1 hyperintense collection in the right hemivagina (blue arrow).

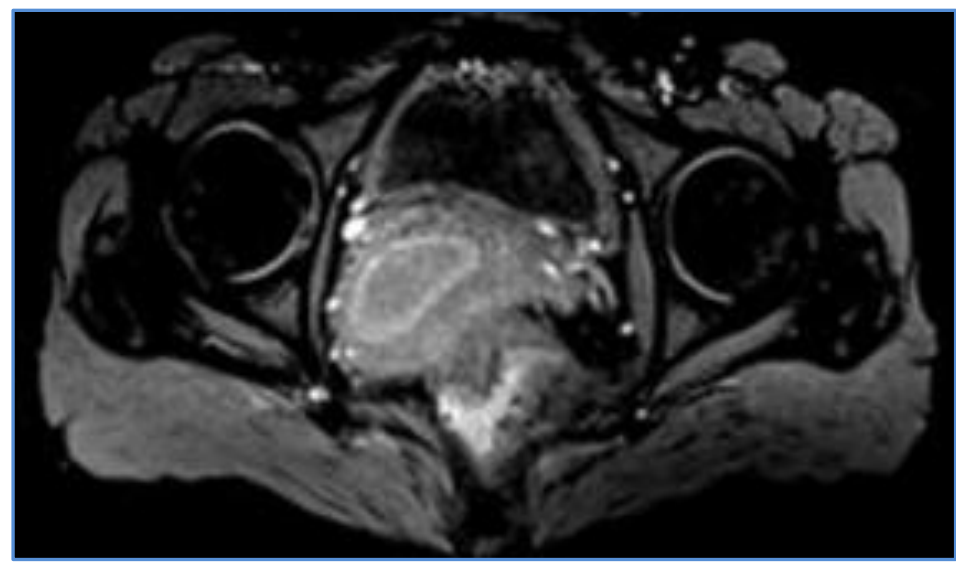

Fig. 3C

\section{REFERENCES:}

1. Herlyn-Werner-Wunderlich syndrome presenting with infertility: Role of MRI in diagnosis. Zohra Ahmad, Ankur Goyal, Chandan J Das, Dipika Deka, and Raju Sharma Indian J Radiol Imaging. 2013 Jul-Sep; 23(3): 243-246.

doi: 10.4103/0971-3026.120283 PMCID: PMC3843333.

2. Herlyn-Werner-Wunderlich syndrome with pregnancy: A rare presentation. Congenit Anom (Kyoto). 2008 Sep; 48 (3):142-3. doi: 10.1111/j.1741-4520.2008.00195.x. Rana R1, Pasrija S, Puri M. PMID: 18778460 [PubMed - indexed for MEDLINE].

3. O'Neill MJ, Yoder IC, Connolly SA et al (1999) Imaging evaluation and classification of developmental anomalies of the female reproductive system with an emphasis on MR imaging. AJR Am J Roentgenol 173:407-416. 
4. Tanaka YO, Kurosaki Y, Kobayashi T et al (1998) Uterus didelphys associated with obstructed hemivagina and ipsilateral renal agenesis: MR findings in seven cases. Abdom Imaging 23:437441.

5. Herlyn-Werner-Wunderlich syndrome: a rare cause of infertility (2009: 2b). Armagan SaracMustafa Kemal Demir. Eur Radiol (2009) 19: 1306-1308 DOI 10.1007/s00330-0081085-8.

6. Yoder IC, Pfister RC (1976) unilateral hematocolpos and ipsilateral renal agenesis: Report of two cases and review of the literature. AJR Am J Roentgenol 127:303-308.

7. Crosby WM, Hill EC. Embryology of the Mullerian duct system. Review of present-day theory. Obstet Gynecol 1962; 20:507-515. [PubMed].

8. Rock JA, Jones HW Jr. The double uterus associated with an obstructed hemivagina and ipsilateral renal agenesis. Am J Obstet Gynecol 1980; 138:339-342. [PubMed].

9. Heinonen PK. Clinical implications of the didelphic uterus: long-term follow-up of 49 cases. Eur J Obstet Gynecol Reprod Biol 2000; 91:183-190. [PubMed].

10. Rock JA, Schlaff WD. The obstetric consequences of uterovaginal anomalies. Fertil Steril 1985; 43:681-692. [PubMed].

11. Moshiri M, Seyal AR, Cruite I, Bhargava P. Herlyn-Werner-Wunderlich syndrome with a partially obstructed hemivagina. Radiology Case Reports. (Online) 2012; 7: 800. Volume 7, Issue 4, 2012. DOI: 10.2484/rcr.v7i4.800.

12. Rare Case of Uterus Didelphis with Full Term Pregnancy in Each Horn Ruchika Garg*, Anita Kwatra**, V. B. Bangal** Pravara Med Rev 2010; 2 (4).

13. Acta Eur Fertil. 1989 May-Jun; 20(3):121-4. Reproductive performance in women with uterus didelphys. Maneschi I ${ }^{1}$, Maneschi F, Parlato M, Fucà G, Incandela S.

14. Green LK, Harris RE. Uterine anomalies; frequency of diagnosis and associated obstetric complications. Obstet Gynecol 1976; 47: 427-42.

\section{AUTHORS:}

1. Priyanka Priyadarshini Baishya

2. Deb Kumar Boruah

3. Chang Hom Thoumoung

4. Dhaval Dhingahi

\section{PARTICULARS OF CONTRIBUTORS:}

1. Registrar, Department of Radio-diagnosis, Assam Medical College, Dibrugarh.

2. Assistant Professor, Department of Radiodiagnosis, Assam Medical College, Dibrugarh.

3. Registrar, Department of Radio-diagnosis, Assam Medical College, Dibrugarh.

FINANCIAL OR OTHER COMPETING INTERESTS: None
4. Post Graduate Trainee, Department of Radiodiagnosis, Assam Medical College, Dibrugarh.

\section{NAME ADDRESS EMAIL ID OF THE CORRESPONDING AUTHOR:}

Dr. Priyanka Priyadarshini Baishya, Department of Radio-diagnosis, Assam Medical College \& Hospital, Dibrugarh-786002, Assam.

E-mail: priyankapriyadarshini1986@gmail.com

Date of Submission: 23/04/2015.

Date of Peer Review: 24/04/2015.

Date of Acceptance: 23/05/2015.

Date of Publishing: 01/06/2015. 